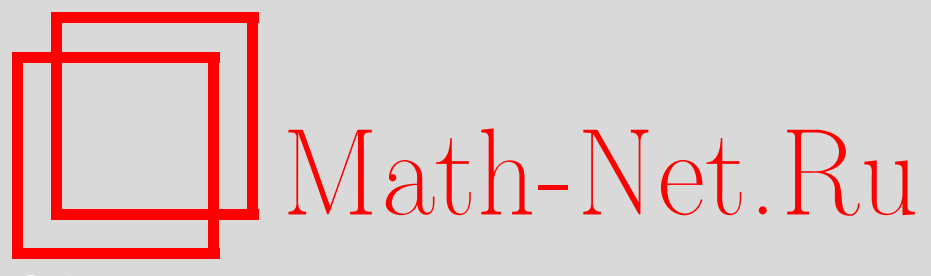

С. Б. Вакарчук, Неравенства типа Джексона и поперечники классов функций в $L_{2}$, Матем. заметки, 2006, том 80, выпуск 1, 11-19

DOI: https://doi.org/10.4213/mzm2774

Использование Общероссийского математического портала Math-Net.Ru подразумевает, что вы прочитали и согласны с пользовательским соглашением http://www . mathnet.ru/rus/agreement

Параметры загрузки:

IP : 3.80 .253 .173

26 апреля 2023 г., 15:52:08

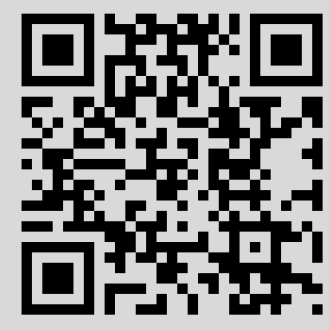




\section{НЕРАВЕНСТВА ТИПА ДЖЕКСОНА И ПОПЕРЕЧНИКИ КЛАССОВ ФУНКЦИЙ В $L_{2}$}

\section{С. Б. Вакарчук}

Точные неравенства типа Джексона, полученные Л. В. Тайковым в пространстве $L_{2}$ и содержащие наилучшее приближение и модуль непрерывности первого порядка, обобщены на модули непрерывности $k$-го порядка $(k=2,3, \ldots)$. Найдены также точные значения $n$-поперечников классов функций $F(k, r, \Phi)$ и $\mathcal{F}_{k}^{r}(h)$, являющихся обобщением рассмотренных Тайковым классов $F(1, r, \Phi)$ и $\mathcal{F}_{1}^{r}(h)$.

Библиография: 14 названий.

1. Через $L_{2}=L_{2}([0,2 \pi])$ обозначим пространство измеримых по Лебегу $2 \pi$-периодических функций, у которых норма

$$
\|f\|=\left\{\frac{1}{\pi} \int_{0}^{2 \pi}|f(x)|^{2} d x\right\}^{1 / 2}<\infty .
$$

Пусть $\mathcal{T}_{n-1}$ есть подпространство, состоящее из всевозможных тригонометрических полиномов порядка $n-1$. Известно, что для произвольной функции $f(x) \in L_{2}$, имеющей разложение в ряд Фурье

$$
f(x) \sim \frac{a_{o}(f)}{2}+\sum_{j=1}^{\infty} a_{j}(f) \cos j x+b_{j}(f) \sin j x,
$$

величина ее наилучшего приближения в метрике $L_{2}$ подпространством $\mathcal{T}_{n-1}$ равна

$$
\begin{aligned}
E_{n-1}(f) & =\inf \left\{\left\|f-T_{n-1}\right\|: T_{n-1}(x) \in \mathcal{T}_{n-1}\right\} \\
& =\left\|f-S_{n-1}(f)\right\|=\left\{\sum_{j=n}^{\infty} \rho_{j}^{2}(f)\right\}^{1 / 2},
\end{aligned}
$$

где $S_{n-1}(f, x)$ есть частная сумма порядка $n-1$ ряда Фурье функции $f(x)$, а $\rho_{j}^{2}(f) \stackrel{\text { df }}{=}$ $a_{j}^{2}(f)+b_{j}^{2}(f)$. Модуль непрерывности $k$-го порядка функции $f(x) \in L_{2}$ обозначим через

$$
\omega_{k}(f, t)=\sup \left\{\left\|\Delta_{h}^{k} f(\cdot)\right\|:|h| \leqslant t\right\},
$$

где $\Delta_{h}^{k} f(x)=\sum_{j=0}^{k}(-1)^{k-j}\left(\begin{array}{c}k \\ j\end{array}\right) f(x+j h)$. Под $L_{2}^{r} \quad\left(r \in \mathbb{Z}_{+}, L_{2}^{0}=L_{2}\right)$ понимаем множество функций $f(x) \in L_{2}$, у которых производные $(r-1)$-го порядка абсолютно непрерывны, а производные $r$-го порядка принадлежат пространству $L_{2}$.

(C) С. Б. ВАКАРчУК, 2006 
При решении задач теории аппроксимации в $L_{2}$, связанных с нахождением точных констант в неравенствах типа Джексона

$$
E_{n-1}(f) \leqslant \chi \cdot n^{-r} \omega_{k}\left(f^{(r)}, \frac{t}{n}\right), \quad t>0,
$$

где $f(x) \in L_{2}^{r} \quad\left(r \in \mathbb{Z}_{+}, f^{(0)}(x) \equiv f(x)\right)$, рассматривались различные экстремальные характеристики, приводящие к уточнению оценок сверху постоянных $\chi$. Для компактного изложения некоторых результатов, полученных ранее, введем следующее обозначение:

$$
\chi_{n, r, k, p, s, m}(\Psi, t) \stackrel{\mathrm{df}}{=} \sup \left\{\frac{n^{2 r-p} E_{n-1}^{2}(f)}{\left(\int_{0}^{t} \omega_{k}^{s}\left(f^{(r)}, x\right) \Psi(x) d x\right)^{m}}: f(x) \in L_{2}^{r}, f(x) \not \equiv \text { const }\right\},
$$

где $n, k \in \mathbb{N} ; r, p \in \mathbb{Z}_{+} ; s, m \in(0, \infty)$. Величины вида (2) в разное время изучали Н. И. Черных [1] $\left(\chi_{n, r, 1,1,2,1}(\Psi, \pi / n)\right.$, где $\Psi(x)=\sin n x ; \chi_{n, 0, k, 0,2,1}(\Psi, 2 \pi / n)$, где $\Psi(x)=\sin (n x / 2)+(\sin n x) / 2)$, Л. В. Тайков [2] $\left(\chi_{n, r, 1,0,2,1}(\Psi, t)\right.$, где $\Psi(x) \equiv 1$; $0<t \leqslant \pi /(2 n))$ и [3] $\left(\chi_{n, r, k, 0,2,1}(\Psi, t)\right.$, где $\left.\Psi(x) \equiv 1 ; 0<t \leqslant \pi / n\right)$, А. А. Лигун [4] $\left(\chi_{n, r, k, 0,2,1}(\Psi, t)\right.$, где $\left.\Psi(x) \geqslant 0 ; 0<t \leqslant \pi / n\right)$, В. В. Шалаев [5] $\left(\chi_{n, r, k, k, 2 / k, k}(\Psi, \pi / n)\right.$, где $\Psi(x)=\sin n x$; при $k=1$ имеем соответствующий результат из [1]), Х. Юссеф [6] $\left(\chi_{n, r, 1,0,2,1}(\Psi, t)\right.$, где $\left.\Psi(x)=\sin (\pi x / t) ; 0<t \leqslant \pi / n\right)$, М. Г. Есмаганбетов [7] $\left(\chi_{n, r, k, 0, s, 2 / s}(\Psi, t)\right.$, где $\Psi(x)=\sin ^{\gamma}(\beta x / t), 0<s \leqslant 2,0 \leqslant \gamma \leqslant r s-1,0<\beta \leqslant \pi$; $0<t \leqslant \pi / n)$.

Экстремальные характеристики, в определенном смысле аналогичные (2), в пространстве $L_{2}$ рассматривались и другими авторами (см., например, [8]-[12]).

Целью данного сообщения является распространение основных результатов работы [2], полученных Тайковым для модуля непрерывности первого порядка, на произвольные модули непрерывности $k$-го порядка $(k=2,3, \ldots)$. Развитие подхода, предложенного автором в [12], позволило решить эту задачу.

Теорема 1. Пустъ $0<t \leqslant \pi /(2 n)$. Тогда для любых чисел $n, k \in \mathbb{N} u r \in \mathbb{Z}_{+}$ справедливы равенства

$$
\chi_{n, r, k, 0,2 / k, k}(1, t)=\left\{\frac{n}{2(n t-\sin n t)}\right\}^{k} .
$$

Eсли $0<t \leqslant \pi / n$, mo

$$
\begin{aligned}
\frac{1}{(n t)^{2 k} n^{2 r}} & \leqslant \sup \left\{\frac{E_{n-1}^{2}(f)}{\omega_{k}^{2}\left(f^{(r)}, t\right)}: f(x) \in L_{2}^{r}, f(x) \not \equiv \text { const }\right\} \\
& \leqslant \frac{1}{n^{2 r}}\left(\frac{1}{(n t)^{2}}+\frac{1}{2}\right)^{k} .
\end{aligned}
$$

2. Прежде чем сформулировать остальные результаты, напомним необходимые понятия и определения. Пусть $\mathbb{B}$ - единичный шар в $L_{2} ; Q$ - выпуклое центральносимметричное подмножество из $L_{2} ; \Lambda_{n} \subset L_{2}-n$-мерное подпространство; $\Lambda^{n} \subset L_{2}$ - подпространство коразмерности $n$; $: L_{2} \rightarrow \Lambda_{n}$ - непрерывный линейный оператор, переводящий элементы пространства $L_{2}$ в $\Lambda_{n} ; \Im^{\perp}: L_{2} \rightarrow \Lambda_{n}-$ непрерывный 
НЕРАВЕНСТВА ТИПА ДЖЕКСОНА И ПОПЕРЕЧНИКИ КЛАССОВ ФУНКЦИЙ В $L_{2}$

оператор линейного проектирования $L_{2}$ на подпространство $\Lambda_{n}$. Величины

$$
\begin{aligned}
b_{n}\left(Q ; L_{2}\right) & =\sup \left\{\sup \left\{\varepsilon>0: \varepsilon \mathbb{B} \cap \Lambda_{n+1} \subset Q\right\}: \Lambda_{n+1} \subset L_{2}\right\}, \\
d_{n}\left(Q ; L_{2}\right) & =\inf \left\{\sup \left\{\inf \left\{\|f-g\|: g \in \Lambda_{n}\right\}: f \in Q\right\}: \Lambda_{n} \subset L_{2}\right\}, \\
\delta_{n}\left(Q ; L_{2}\right) & =\inf \left\{\inf \left\{\sup \{\|f-\Im f\|: f \in Q\}: \Im L_{2} \subset \Lambda_{n}\right\}: \Lambda_{n} \subset L_{2}\right\}, \\
d^{n}\left(Q ; L_{2}\right) & =\inf \left\{\sup \left\{\|f\|: f \in Q \cap \Lambda^{n}\right\}: \Lambda^{n} \subset L_{2}\right\}, \\
\Pi_{n}\left(Q ; L_{2}\right) & =\inf \left\{\inf \left\{\sup \left\{\left\|f-\Im^{\perp} f\right\|: f \in Q\right\}: \Im^{\perp} L_{2} \subset \Lambda_{n}\right\}: \Lambda_{n} \subset L_{2}\right\}
\end{aligned}
$$

называют соответственно бернштейновским, колмогоровским, линейным, гельфандовским, проекиионным $n$-поперечниками. Так как $L_{2}$ является гильбертовым пространством, справедливы следующие соотношения между перечисленными величинами (см., например, [13], [14]):

$$
b_{n}\left(Q ; L_{2}\right) \leqslant d^{n}\left(Q ; L_{2}\right) \leqslant d_{n}\left(Q ; L_{2}\right)=\delta_{n}\left(Q ; L_{2}\right)=\Pi_{n}\left(Q ; L_{2}\right) .
$$

Всюду далее под $\Phi(t), 0 \leqslant t<\infty$, понимаем непрерывную монотонно возрастающую функцию, обращающуюся в нуль в точке $t=0$. Рассмотрим классы

$$
F(k, r, \Phi) \stackrel{\mathrm{df}}{=}\left\{f(x) \in L_{2}^{r}: \int_{0}^{t} \omega_{k}^{2 / k}\left(f^{(r)}, \tau\right) d \tau \leqslant \Phi(t) \forall t>0\right\},
$$

где $k, r \in \mathbb{N}$. Тайков показал [2], что при выполнении ограничений

$$
\frac{\Phi(t)}{\Phi(\pi /(2 n))} \geqslant \frac{2}{\pi-2} \begin{cases}n t-\sin n t, & \text { если } 0<t \leqslant \pi / n, \\ 2 n t-\pi, & \text { если } t \geqslant \pi / n,\end{cases}
$$

справедливы равенства

$$
d_{2 n-1}\left(F(1, r, \Phi) ; L_{2}\right)=n^{-r}\left\{\frac{n}{\pi-2} \Phi\left(\frac{\pi}{2 n}\right)\right\}^{1 / 2} \quad \forall n \in \mathbb{N} .
$$

Функция $\Phi_{*}(t)=t^{\pi /(\pi-2)}$ является примером мажоранты, удовлетворяющей условиям (6).

Для $r, k \in \mathbb{N}$ и $h>0$ полагаем

$$
\mathcal{F}_{k}^{r}(h) \stackrel{\mathrm{df}}{=}\left\{f(x) \in L_{2}^{r}: \int_{0}^{h} \omega_{k}^{2 / k}\left(f^{(r)}, \tau\right) d \tau \leqslant 1\right\} .
$$

В [2] было показано, что если натуральное число $n$ удовлетворяет условию $n h \leqslant \pi / 2$, то

$$
d_{2 n-1}\left(\mathcal{F}_{1}^{r}(h), L_{2}\right)=n^{-r}\left\{\frac{n}{2(n h-\sin n h)}\right\}^{1 / 2} .
$$

Следующие теоремы обобщают результаты $(7)$ и $(8)$ на классы $F(k, r, \Phi)$ и $\mathcal{F}_{k}^{r}(h)$, где $k=2,3, \ldots$, соответственно.

Теорема 2. Если мажоранта $\Phi(t)$ удовлетворяет ограничениям (6), то для любых натуральных чисел $n, k u r \in \mathbb{Z}_{+}$имеют место следующие равенства:

$$
p_{2 n-1}\left(F(k, r, \Phi) ; L_{2}\right)=p_{2 n}\left(F(k, r, \Phi) ; L_{2}\right)=n^{-r}\left\{\frac{n}{\pi-2} \Phi\left(\frac{\pi}{2 n}\right)\right\}^{k / 2},
$$

где $p_{m}(\cdot)$ - любой из перечисленных выще $m$-поперечников. 
СлеДствиЕ 1. Для любых натуральных чисел $n, k u r \in \mathbb{Z}_{+}$справедливы равенства

$$
p_{2 n-1}\left(F\left(k, r, \Phi_{*}\right) ; L_{2}\right)=p_{2 n}\left(F\left(k, r, \Phi_{*}\right) ; L_{2}\right)=\frac{n^{-r-k /(\pi-2)}}{(\pi-2)^{k / 2}}\left(\frac{\pi}{2}\right)^{\pi k /(2(\pi-2))},
$$

где $p_{m}(\cdot)$ - любой из m-поперечников, рассмотреннъх ранее.

ТеОрема 3. Пусть $k, r, n \in \mathbb{N}, h>0$ и выполнено условие $n h \leqslant \pi / 2$. Тогда

$$
p_{2 n-1}\left(\mathcal{F}_{k}^{r}(h), L_{2}\right)=p_{2 n}\left(\mathcal{F}_{k}^{r}(h), L_{2}\right)=n^{-r}\left\{\frac{n}{2(n h-\sin n h)}\right\}^{k / 2},
$$

где $p_{m}(\cdot)$ - любой из рассмотренных ранее m-поперечников.

3. Доказательство теоремы 1. В силу определения гильбертова пространства $L_{2}$ и свойств тригонометрической системы, для произвольной функции $f(x) \in$ $L_{2}$ запишем

$$
\left\|\Delta_{h}^{k} f^{(r)}(\cdot)\right\|^{2}=2^{k} \sum_{j=1}^{\infty} j^{2 r} \rho_{j}^{2}(f)(1-\cos j h)^{k} .
$$

В силу (1) получим

$$
E_{n-1}^{2}(f)-\sum_{j=n}^{\infty} \rho_{j}^{2}(f) \cos j h=\sum_{j=n}^{\infty} \rho_{j}^{2-2 / k}(f) \rho_{j}^{2 / k}(f)(1-\cos j h) .
$$

Применив к правой части равенства (12) рассуждения из работы [5], а именно, используя неравенство Гёльдера для числовых рядов, а также учитывая (11) и определение модуля непрерывности $k$-го порядка, имеем

$$
\begin{aligned}
E_{n-1}^{2}(f)-\sum_{j=n}^{\infty} \rho_{j}^{2}(f) \cos j h & \leqslant E_{n-1}^{2-2 / k}(f)\left\{\frac{1}{n^{2 r}} \sum_{j=n}^{\infty} j^{2 r} \rho_{j}^{2}(f)(1-\cos j h)^{k}\right\}^{1 / k} \\
& \leqslant E_{n-1}^{2-2 / k}(f) \frac{\omega_{k}^{2 / k}\left(f^{(r)}, h\right)}{2 n^{2 r / k}} .
\end{aligned}
$$

Проинтегрировав соотношение (13) по $h$ в пределах от 0 до $t$ и умножив затем обе части полученного неравенства на величину $1 / t$, запишем

$$
E_{n-1}^{2}(f) \leqslant \sum_{j=n}^{\infty} \rho_{j}^{2}(f) \frac{\sin j t}{j t}+\frac{E_{n-1}^{2-2 / k}(f)}{2 n^{2 r / k} t} \int_{0}^{t} \omega_{k}^{2 / k}\left(f^{(r)}, h\right) d h .
$$

Поскольку при $0<n t \leqslant \pi / 2$ справедливо равенство [2]

$$
\max _{n t \leqslant \tau} \frac{|\sin \tau|}{\tau}=\frac{\sin n t}{n t},
$$

из (14), (15) и (1) имеем

$$
E_{n-1}^{2}(f) \leqslant \frac{\sin n t}{n t} E_{n-1}^{2}(f)+\frac{E_{n-1}^{2-2 / k}(f)}{2 n^{2 r / k} t} \int_{0}^{t} \omega_{k}^{2 / k}\left(f^{(r)}, h\right) d h .
$$


НЕРАВЕНСТВА ТИПА ДЖЕКСОНА И ПОПЕРЕЧНИКИ КЛАССОВ ФУНКЦИЙ В $L_{2}$

Из (16) следует оценка сверху

$$
E_{n-1}^{2}(f) \leqslant n^{-2 r}\left\{\frac{n}{2(n t-\sin n t)}\right\}^{k}\left(\int_{0}^{t} \omega_{k}^{2 / k}\left(f^{(r)}, h\right) d h\right)^{k} .
$$

Используя (2) и (17), запишем

$$
\chi_{n, r, k, 0,2 / k, k}(1, t) \leqslant\left\{\frac{n}{2(n t-\sin n t)}\right\}^{k} .
$$

Для получения оценки снизу рассмотрим в $L_{2}^{r}$ функцию $f_{0}(x) \stackrel{\mathrm{df}}{=} \cos n x$. Поскольку $E_{n-1}\left(f_{0}\right)=1$ и

$$
\omega_{k}\left(f_{0}^{(r)}, h\right)=2^{k / 2} n^{r}(1-\cos n h)^{k / 2},
$$

где $0<h \leqslant \pi / n$, то с учетом (2) имеем

$$
\chi_{n, r, k, 0,2 / k, k}(1, t) \geqslant \frac{n^{2 r} E_{n-1}^{2}\left(f_{0}\right)}{\left(\int_{0}^{t} \omega_{k}^{2 / k}\left(f_{0}^{(r)}, h\right) d h\right)^{k}}=\left\{\frac{n}{2(n t-\sin n t)}\right\}^{k} .
$$

Сопоставив оценки сверху (18) и снизу (20), получим требуемое равенство (3).

Перейдем к доказательству соотношения (4). Для этого проинтегрируем дважды неравенство (13) - вначале по переменной $h$ в пределах от 0 до $\tau$, а затем по переменной $\tau$ в пределах от 0 до $t$. В результате этого будем иметь

$$
\begin{gathered}
\frac{t^{2}}{2} E_{n-1}^{2}(f) \leqslant \sum_{j=n}^{\infty} \frac{1}{j^{2}} \rho_{j}^{2-2 / k}(f) \rho_{j}^{2 / k}(f)(1-\cos j t) \\
+\frac{E_{n-1}^{2-2 / k}(f)}{2 n^{2 r / k}} \int_{0}^{t} \int_{0}^{\tau} \omega_{k}^{2 / k}\left(f^{(r)}, h\right) d h d \tau .
\end{gathered}
$$

Применив, с учетом (1), неравенство Гёльдера к числовому ряду в соотношении (21), а затем произведя в повторном интеграле процедуру интегрирования по частям для более рациональной формы его записи, получим

$$
\begin{aligned}
\frac{t^{2}}{2} E_{n-1}^{2}(f) \leqslant & \frac{E_{n-1}^{2-2 / k}(f)}{n^{2}}\left\{\frac{1}{n^{2 r}} \sum_{j=n}^{\infty} j^{2 r} \rho_{j}^{2}(f)(1-\cos j t)^{k}\right\}^{1 / k} \\
& +\frac{E_{n-1}^{2-2 / k}(f)}{2 n^{2 r / k}} \int_{0}^{t}(t-\tau) \omega_{k}^{2 / k}\left(f^{(r)}, \tau\right) d \tau \\
\leqslant & \frac{E_{n-1}^{2-2 / k}(f)}{2 n^{2+2 r / k}}\left\{\omega_{k}^{2 / k}\left(f^{(r)}, t\right)+n^{2} \int_{0}^{t}(t-\tau) \omega_{k}^{2 / k}\left(f^{(r)}, \tau\right) d \tau\right\} .
\end{aligned}
$$

Отсюда имеем

$$
t^{2 k} E_{n-1}^{2}(f) \leqslant \frac{1}{n^{2(r+k)}}\left\{\omega_{k}^{2 / k}\left(f^{(r)}, t\right)+n^{2} \int_{0}^{t}(t-\tau) \omega_{k}^{2 / k}\left(f^{(r)}, \tau\right) d \tau\right\}^{k} .
$$

Для произвольной функции $f(x) \in L_{2}^{r}, f(x) \not \equiv$ const, из (22) получим

$$
t^{2 k} E_{n-1}^{2}(f) \leqslant \frac{1}{n^{2(r+k)}} \omega_{k}^{2}\left(f^{(r)}, t\right)\left(1+\frac{(n t)^{2}}{2}\right)^{k} .
$$


Следовательно,

$$
\frac{E_{n-1}^{2}(f)}{\omega_{k}^{2}\left(f^{(r)}, t\right)} \leqslant \frac{1}{n^{2 r}}\left(\frac{1}{(n t)^{2}}+\frac{1}{2}\right)^{k} .
$$

Для рассмотренной ранее функции $f_{0}(x)=\cos n x$ при $0<t \leqslant \pi / n$ в силу (19) имеем

$$
\omega_{k}^{2}\left(f_{0}^{(r)}, t\right)=2^{2 k} n^{2 r} \sin ^{2 k}\left(\frac{n t}{2}\right) \leqslant n^{2 r}(n t)^{2 k} .
$$

Тогда

$$
\frac{E_{n-1}^{2}\left(f_{0}\right)}{\omega_{k}^{2}\left(f_{0}^{(r)}, t\right)} \geqslant \frac{1}{(n t)^{2 k} n^{2 r}} .
$$

Двойное неравенство (4), обобщающее соответствующий результат Тайкова [2] на модуль непрерывности $k$-го порядка $\omega_{k}\left(f^{(r)}, t\right), k=2,3, \ldots$, получаем из $(23),(24)$, чем и завершаем доказательство теоремы 1.

4. Доказательство теоремы 2. Полагая в (17) $t=\pi /(2 n)$, для произвольной функции $f(x) \in F(k, r, \Phi)$ запишем

$$
E_{n-1}(f) \leqslant n^{-r}\left\{\frac{n}{\pi-2} \Phi\left(\frac{\pi}{2 n}\right)\right\}^{k / 2} .
$$

Используя (5) и (25), получим оценки сверху для рассмотренных в п. $2 n$-поперечников

$$
\begin{aligned}
p_{2 n}\left(F(k, r, \Phi) ; L_{2}\right) & \leqslant p_{2 n-1}\left(F(k, r, \Phi) ; L_{2}\right) \leqslant d_{2 n-1}\left(F(k, r, \Phi) ; L_{2}\right) \\
& \leqslant E_{n-1}(F(k, r, \Phi)) \leqslant n^{-r}\left\{\frac{n}{\pi-2} \Phi\left(\frac{\pi}{2 n}\right)\right\}^{k / 2},
\end{aligned}
$$

где $E_{n-1}(\mathcal{M})=\sup \left\{E_{n-1}(f): f(x) \in \mathcal{M}\right\}, \mathcal{M}-$ произвольный класс функций из $L_{2}$.

Для получения оценок снизу перечисленных в п. $2 n$-поперечников рассмотрим в $\mathcal{T}_{n} \cap L_{2}$ шар

$$
\mathbb{B}_{2 n+1} \stackrel{\text { df }}{=}\left\{T_{n}(x) \in \mathcal{T}_{n}:\left\|T_{n}\right\| \leqslant n^{-r}\left(\frac{n}{\pi-2} \Phi\left(\frac{\pi}{2 n}\right)\right)^{k / 2}\right\}
$$

и покажем его принадлежность классу $F(k, r, \Phi)$. Для этого нам понадобится неравенство [3]

$$
\omega_{k}^{2}\left(T_{n}^{(r)}, t\right) \leqslant 2^{k} n^{2 r}(1-\cos n t)_{*}^{k}\left\|T_{n}\right\|^{2},
$$

где $(1-\cos n t)_{*} \stackrel{\text { df }}{=}\{1-\cos n t$, если $0 \leqslant t \leqslant \pi / n ; 2$, если $t \geqslant \pi / n\} ; T_{n}(x)-$ произвольный тригонометрический полином из подпространства $\mathcal{T}_{n}$.

Пусть $0<t \leqslant \pi / n$. Используя определение класса $F(k, r, \Phi)$, первое неравенство из ограничения (6) и соотношение $(27)$, для любого полинома $T_{n}(x) \in \mathbb{B}_{2 n+1}$ получим

$$
\begin{aligned}
\int_{0}^{t} \omega_{k}^{2 / k}\left(T_{n}^{(r)}, \tau\right) d \tau & \leqslant 2 n^{2 r / k}\left\|T_{n}\right\|^{2 / k} \int_{0}^{t}(1-\cos n \tau) d \tau \\
& \leqslant \frac{2}{\pi-2}(n t-\sin n t) \Phi\left(\frac{\pi}{2 n}\right) \leqslant \Phi(t)
\end{aligned}
$$


НЕРАВЕНСТВА ТИПА ДЖЕКСОНА И ПОПЕРЕЧНИКИ КЛАССОВ ФУНКЦИЙ В $L_{2}$

Пусть $\pi / n \leqslant t$. На основании аналогичных соображений и второго неравенства из ограничения (6) для произвольного полинома $T_{n}(x) \in \mathbb{B}_{2 n+1}$ запишем

$$
\begin{aligned}
\int_{0}^{t} \omega_{k}^{2 / k}\left(T_{n}^{(r)}, \tau\right) d \tau & \leqslant 2 n^{2 r / k}\left\|T_{n}\right\|^{2 / k}\left(2 t-\frac{\pi}{n}\right) \\
& \leqslant \frac{2}{\pi-2}(2 n t-\pi) \Phi\left(\frac{\pi}{2 n}\right) \leqslant \Phi(t) .
\end{aligned}
$$

Из (28), $(29)$ следует включение $\mathbb{B}_{2 n+1} \subset F(k, r, \Phi)$. Используя (6) и определение бернштейновского $n$-поперечника, имеем

$$
\begin{aligned}
p_{2 n}\left(F(k, r, \Phi) ; L_{2}\right) & \geqslant b_{2 n}\left(F(k, r, \Phi) ; L_{2}\right) \geqslant b_{2 n}\left(\mathbb{B}_{2 n+1}, L_{2}\right) \\
& \geqslant n^{-r}\left\{\frac{n}{\pi-2} \Phi\left(\frac{\pi}{2 n}\right)\right\}^{k / 2} .
\end{aligned}
$$

Сопоставляя неравенства (26) и (30), получим соотношения (9), чем и завершим доказательство теоремы 2.

СлЕДСТвИЕ 2. Если выполнены условия теоремы 2, то имеют место следующие равенства:

$$
\begin{aligned}
\sup \left\{\left|a_{n}(f)\right|: f(x) \in F(k, r, \Phi)\right\} & =\sup \left\{\left|b_{n}(f)\right|: f(x) \in F(k, r, \Phi)\right\} \\
& =n^{-r}\left\{\frac{n}{\pi-2} \Phi\left(\frac{\pi}{2 n}\right)\right\}^{k / 2} \quad \forall n \in \mathbb{N} .
\end{aligned}
$$

ДокАЗАТЕЛЬСтво. Не уменьшая общности, проведем рассуждения для коэффициентов $b_{n}(f)$. Учитывая, что

$$
b_{n}(f)=\frac{1}{\pi} \int_{0}^{2 \pi} f(x) \sin n x d x=\frac{1}{\pi} \int_{0}^{2 \pi}\left[f(x)-S_{n-1}(f, x)\right] \sin n x d x
$$

и используя неравенство Коши-Буняковского, а также (1) и (26), получим оценку сверху

$$
\sup \left\{\left|b_{n}(f)\right|: f(x) \in F(k, r, \Phi)\right\} \leqslant E_{n-1}(F(k, r, \Phi)) \leqslant n^{-r}\left\{\frac{n}{\pi-2} \Phi\left(\frac{\pi}{2 n}\right)\right\}^{k / 2} .
$$

Для нахождения оценки снизу рассмотрим функцию

$$
f_{1}(x) \stackrel{\mathrm{df}}{=} n^{-r}\left\{\frac{n}{\pi-2} \Phi\left(\frac{\pi}{2 n}\right)\right\}^{k / 2} \sin n x .
$$

Из доказательства теоремы 2 следует, что $f_{1}(x)$ принадлежит шару $\mathbb{B}_{2 n+1}$, а значит является элементом класса $F(k, r, \Phi)$. Тогда

$$
\sup \left\{\left|b_{n}(f)\right|: f(x) \in F(k, r, \Phi)\right\} \geqslant\left|b_{n}\left(f_{1}\right)\right|=n^{-r}\left\{\frac{n}{\pi-2} \Phi\left(\frac{\pi}{2 n}\right)\right\}^{k / 2} .
$$

Сравнив оценки сверху и снизу, получаем

$$
\sup \left\{\left|b_{n}(f)\right|: f(x) \in F(k, r, \Phi)\right\}=n^{-r}\left\{\frac{n}{\pi-2} \Phi\left(\frac{\pi}{2 n}\right)\right\}^{k / 2}
$$

Следствие 2 доказано. 
5. Доказательство теоремы 3. Используя определение класса $\mathcal{F}_{k}^{r}(h)$, а также соотношения (5) и (17), имеем

$$
\begin{aligned}
p_{2 n}\left(\mathcal{F}_{k}^{r}(h), L_{2}\right) & \leqslant p_{2 n-1}\left(\mathcal{F}_{k}^{r}(h), L_{2}\right) \leqslant d_{2 n-1}\left(\mathcal{F}_{k}^{r}(h), L_{2}\right) \\
& \leqslant E_{n-1}\left(\mathcal{F}_{k}^{r}(h)\right) \leqslant n^{-r}\left\{\frac{n}{2(n h-\sin n h)}\right\}^{k / 2} .
\end{aligned}
$$

Для нахождения оценок снизу $n$-поперечников класса $\mathcal{F}_{k}^{r}(h)$ рассмотрим в $\mathcal{T}_{n} \cap L_{2}$ шар

$$
\widetilde{\mathbb{B}}_{2 n+1} \stackrel{\text { df }}{=}\left\{T_{n}(x) \in \mathcal{T}_{n}:\left\|T_{n}\right\| \leqslant n^{-r}\left(\frac{n}{2(n h-\sin n h)}\right)^{k / 2}\right\} .
$$

Для произвольного полинома $T_{n}(x) \in \widetilde{\mathbb{B}}_{2 n+1}$ при $0<n h \leqslant \pi / 2$ из $(27)$ получим

$$
\int_{0}^{h} \omega_{k}^{2 / k}\left(T_{n}^{(r)}, \tau\right) d \tau \leqslant 2 n^{2 r / k}\left\|T_{n}\right\|^{2 / k}\left(h-\frac{1}{n} \sin n h\right) \leqslant 1
$$

Учитывая определение класса $\mathcal{F}_{k}^{r}(h)$ и неравенство $(32)$, имеем $\widetilde{\mathbb{B}}_{2 n+1} \subset \mathcal{F}_{k}^{r}(h)$. Используя (5) и определение бернштейновского $n$-поперечника, запишем

$$
p_{2 n}\left(\mathcal{F}_{k}^{r}(h), L_{2}\right) \geqslant b_{2 n}\left(\mathcal{F}_{k}^{r}(h), L_{2}\right) \geqslant b_{2 n}\left(\widetilde{\mathbb{B}}_{2 n+1}, L_{2}\right) \geqslant n^{-r}\left\{\frac{n}{2(n h-\sin n h)}\right\}^{k / 2} .
$$

Требуемые равенства (10) следуют из сравнения между собой соотношений (31) и $(33)$, чем и завершается доказательство теоремы 3.

СлЕДСтвиЕ 3. Пусть выполнены условия теоремы 3. Тогда

$$
\sup \left\{\left|a_{n}(f)\right|: f(x) \in \mathcal{F}_{k}^{r}(h)\right\}=\sup \left\{\left|b_{n}(f)\right|: f(x) \in \mathcal{F}_{k}^{r}(h)\right\}=n^{-r}\left\{\frac{n}{2(n h-\sin n h)}\right\}^{k / 2},
$$

где натуральное число $n$ удовлетворяет неравенству $n \leqslant \pi /(2 h)$.

Доказательство данного утверждения не приводится, поскольку повторяет рассуждения, имевшие место при получении следствия 2.

\section{СПИСОК ЦИТИРОВАННОЙ ЛИТЕРАТУРЫ}

[1] Н. И. Черных, "О наилучшем приближении периодических функций тригонометрическими полиномами в $L_{2}$ ", Матем. заметки, 2:5 (1967), 513-522.

[2] Л. В. Тайков, "Неравенства, содержащие наилучшие приближения и модуль непрерывности функций из $L_{2} "$ ", Матем. заметки, 20:3 (1976), 433-438.

[3] Л.В. Тайков, "Структурные и конструктивные характеристики функций из $L_{2}$ ", Матем. заметки, 25:2 (1979), 217-223.

[4] А. А. Лигун, "Некоторые неравенства между наилучшими приближениями и модулями непрерывности в пространстве $L_{2}$ ", Матем. заметки, 24:6 (1978), 785-792.

[5] В. В. Шалаев, "О поперечниках в $L_{2}$ классов дифференцируемых функций, определяемых модулями непрерывности высших порядков", Укр. матем. ж., 43:1 (1991), $125-129$.

[6] Х. Юссеф, "О наилучших приближениях функций и значениях поперечников классов функций в $L_{2}$ ", Применение функиионального анализа в теории приближений, Калининский гос. ун-т, Калинин, 1988, 100-114. 
[7] М. Г. Есмаганбетов, "Поперечники классов из $L_{2}[0,2 \pi]$ и минимизация точных констант в неравенствах типа Джексона", Матем. заметки, 65:6 (1999), 816-820.

[8] С. Б. Вакарчук, "О наилучших полиномиальных приближениях в $L_{2}$ некоторых классов $2 \pi$-периодических функций и точных значениях их $n$-поперечников”, Maтем. заметки, 70:3 (2001), 334-345.

[9] С.Б. Вакарчук, "О наилучших полиномиальных приближениях $2 \pi$-периодических функций и точных значениях $n$-поперечников функциональных классов в пространстве $L_{2} "$ Укр. матем. ж., 54:12 (2002), 1603-1615.

[10] С.Н. Васильев, "Точное неравенство Джексона-Стечкина в $L_{2}$ с модулем непрерывности, порожденным произвольным конечно-разностным оператором с постоянными коэффициентами", Докл. РАН, 385:1 (2002), 11-14.

[11] А.И. Степанец, А.С. Сердюк, "Прямые и обратные теоремы теории приближения функций в пространстве $S^{p ",}$ Укр. матем. ж., 54:1 (2002), 106-124.

[12] С. Б. Вакарчук, А. Н. Щитов, "Наилучшие полиномиальные приближения в $L_{2}$ и поперечники некоторых классов функций”, Укр. матем. ж., 56:11 (2004), 1458-1466.

[13] В. М. Тихомиров, Некоторые вопросы теории приближений, МГУ, М., 1976.

[14] A. Pinkus, n-Widths in Approximation Theory, Springer, Berlin, 1985.

\section{С. Б. Вакарчук}

Академия таможенной службы Украины

г. Днепропетровск

E-mail: academy@amsu.dnp.ukrpack.net
Поступила в редакцию 09.03.2005

Исправленный вариант 25.12 .2005 